\title{
Brown's Atlas of Regional Anesthesia, 6th Edition
}

\author{
Ehab Farag, Loran Mounir-Soliman. Elsevier, Philadelphia, 2020; 312 pages; ISBN: \\ 978-0-323654357
}

\author{
Carole-Anne Potvin, MD · Derek Dillane, MD, FRCPC (1)
}

Received: 24 November 2020/Accepted: 25 November 2020/Published online: 6 January 2021

(C) Canadian Anesthesiologists' Society 2021

First published in 1992, Brown's Atlas of Regional Anesthesia is considered a canonical reference in regional anesthesia. This latest edition, the sixth, is the result of an iterative process in the intervening period that has seen a single author publication evolve into a multi-author endeavor that is available in print and electronic formats, with accompanying instructional videos.

The most striking change from previous editions is the elimination of a hitherto recurring section on chronic pain and related blocks. Except for a chapter on stellate ganglion blockade, the sixth edition is devoted exclusively to regional anesthesia techniques. The primary editors of the fifth edition, Ehab Farag and Loran Mounir-Soliman, remain at the helm for this latest edition. The list of contributing authors, however, has more than doubled (to 20), most of whom are colleagues of Farag and Mounir-Soliman at the Cleveland Clinic.

The book is conveniently divided into nine sections. The introductory section includes new chapters on pediatric local anesthetic pharmacology, and equipment and ultrasound. Subsequent sections on upper extremity, lower extremity, head and neck, airway, truncal, and neuraxial blocks have been revised to include chapters on techniques newly described since the 5th edition was published in 2017. In a similar vein, there is an expanded section on ultrasound-guided pediatric regional anesthesia and a welcome new section on obstetric regional anesthesia.

For the most part, there is a consistency to the structure and content throughout the main sections. Many chapters start with key point information for a specific block, followed by a high-level commentary on anatomy,

C.-A. Potvin, MD · D. Dillane, MD, FRCPC ( $\square)$

University of Alberta, Edmonton, AB, Canada

e-mail: dillane@ualberta.ca patient selection, and pharmacologic choice. The text remains faithful to its provenance in many chapters through detailed instruction on approaches which might be considered of more historical than practical significance. This is occasionally at the expense of more detailed descriptions of commonly utilized ultrasound-guided approaches and accompanying ultrasound images. All relevant chapters contain well organized descriptions of ultrasound techniques, with specific attention given to sonoanatomy, scanning, and needling technique. Though the book is illustrated resplendently throughout with color line drawings, the reader would likely benefit from a greater selection of ultrasound images of a similar scale to the illustrations. Many chapters conclude with a "pearls" section which contain countless indispensable observations from the bedside.

Though the specialty of regional anesthesia is constantly evolving and the number of "must know" blocks seemingly ever expanding, this book only occasionally deviates from the time-tested stalwarts. It would be unfair to say that the primary editors are oblivious to progress, rather they curate in a prudent manner, respectful of the legacy to which they have been entrusted. Hence, readers who are new to this reference, or indeed regional anesthesia, will be rewarded with all of the foundational elements required to build a solid substratum of knowledge. Returning readers will find few changes to the first five sections of this book compared to the previous edition. An updated list of references or suggested reading, regrettably short or missing entirely from many chapters, might be considered for future editions. A new addition to the 6th edition, a chapter on ultrasound guided fascia iliaca and inguinal region blocks encapsulates our criticisms of the book as a whole. For example, while the description of the au courant suprainguinal fasica iliaca block is described in great detail, it is only supported by one small ultrasound image and no references. 
Of interest to owners of previous editions, and commensurate with recent developments in interfascial planar blocks, most new content in the 6th edition can be found in the section on truncal blocks. Arguably containing the strongest chapters, this is where the reader will find the most complete array of accompanying images and videos. There are new chapters on pectoralis and pecto-intercostal, serratus anterior, intercostal, erector spinae plane and rectus sheath blockade. Chapters pertaining to paravertebral and transversus abdominis plane (TAP) blockade, and all chapters in the section on neuraxial blockade, have been minimally revised to include an opening box highlighting key points. Ultrasound images are conspicuous by their absence in the chapter on ultrasound-assisted neuraxial blocks. However, the entire section to which the chapter belongs is an indulgence for the traditionalist.

The pediatric regional anesthesia section includes concise chapters on the basic elements of blocks that are a regular part of pediatric anesthesia practice - i.e., caudal, ilioinguinal and iliohypogastric, superficial cervical plexus, pudendal, and continuous paravertebral blockade. Finally, a new section on regional techniques during pregnancy and delivery contains an overview of the physiological changes during pregnancy and how such changes can affect neuraxial block placement in the parturient. This concluding chapter, more verbose than preceding chapters, is an erudite mix of the practical and the conceptual. In this case, last is certainly not least.
Brown's Atlas of Regional Anesthesia, $6^{\text {th }}$ Edition is also available as a multimedia version accessible via desktop computer, tablet, or smartphone, and it can be downloaded for offline use. The electronic book contains a complete version of the original text, as well as videos of ultrasoundguided blocks. The authors are explicit in recommending that the videos be used in conjunction with the text. Unfortunately, the lack of references to videos in the text makes that match-up challenging.

In summary, Brown's Atlas of Regional Anesthesia, $6^{\text {th }}$ Edition, offers a broad overview of regional anesthesia techniques with meticulous descriptions of landmark approaches and splendid anatomical illustrations. Even though there is an apparent desire on the part of the editors to embrace ultrasound guidance, the dearth of high-quality ultrasound images impedes progress in this direction. As a result, the textbook may be less suitable for learners who wish to focus exclusively on ultrasound-guided techniques. For "ultrasound curious" readers who developed their regional anesthesia skills in the era of landmark-based nerve blockade, this book is highly recommended.

Disclosures None.

Funding statement None.

Editorial responsibility This submission was handled by Dr. Hilary P. Grocott, Editor-in-Chief, Canadian Journal of Anesthesia.

Publisher's Note Springer Nature remains neutral with regard to jurisdictional claims in published maps and institutional affiliations. 